\title{
Fever, abdominal pain, and lymphocytic ascites in a young immigrant from Western Africa
}

\author{
Antonio Faraone ${ }^{1 *}$, Domenico Tomasi ${ }^{2}$ and Alberto Fortini ${ }^{1}$ \\ ${ }^{1}$ Internal Medicine Unit, San Giovanni di Dio Hospital, Florence, Italy \\ ${ }^{2}$ Radiology Unit, San Giovanni di Dio Hospital, Florence, Italy
}

\begin{abstract}
Tuberculous peritonitis is an uncommon disease in countries with low tuberculosis (TB) incidence, most often affecting non-white race, foreign-born individuals. We describe a case of TB with peritoneal involvement in a 32-year-old man immigrated to Italy from Burkina Faso, who presented with a history of fever, malaise, abdominal pain and abdominal swelling. Due to its nonspecific clinical presentation and paucibacillary nature, diagnosis of tuberculous peritonitis can be challenging and requires a high index of suspicion. This report highlights the diagnostic challenges posed by tuberculous peritonitis and emphasizes the importance of imaging (computed tomography, CT) in identifying typical findings, and the value of histological examination of tissue specimens from peritoneum or any site of suspected $\mathrm{TB}$ as a tool for diagnosis confirmation.
\end{abstract}

\section{Learning points}

- Tuberculous peritonitis is a rare disease in western countries and should be suspected in patients at risk for TB who present with fever, abdominal pain, lymphocytic ascites.

- Ascites, thickening and nodules of peritoneum and omentum (omental cake) are the commonest CT imaging findings.

- The yield of microscopic examination, PCR and culture testing for Mycobacterium tuberculosis on ascitic fluid is quite low, and confirmation of diagnosis usually relies on microbiological or histological examination of biopsy specimen from peritoneum or any other site of suspected TB infection.

\section{Case report}

A 32-year-old man from Burkina Faso, who had immigrated to Italy 3 years earlier, was admitted to the internal medicine ward of a medium-size acute-care hospital with a 3-week history of fever, fatigue, weight loss, abdominal pain, and abdominal swelling. The patient was a moderate alcohol consumer, and his past medical history was notable for sickle cell disease. Vital signs were remarkable for body temperature of $38.7^{\circ} \mathrm{C}$, arterial blood pressure of $100 / 70 \mathrm{mmHg}$, heart rate of 100 beats per minute. Oxygen saturation was $98 \%$ on ambient air. At physical examination, the patient was alert and oriented, his abdomen was distended and diffusely tender to palpation, with shifting dullness on percussion, breath sounds over the right lung base were slightly diminished. No heart murmurs, leg swelling, or enlarged lymph nodes were detected. Laboratory tests showed moderate anemia (hemoglobin $8.9 \mathrm{~g} / \mathrm{dL}$ ), a high level of C-reactive protein (CRP, 9.4 $\mathrm{mg} / \mathrm{dL}$ ), a slight increase of lactic dehydrogenase (LDH) and aspartate transaminase (AST), normal levels of bilirubin and haptoglobin (Table 1). QuantiFERON-TB Gold test was negative. Serologic assay for HIV and HCV were negative; testing for HBV, revealed a previous infection (HBsAg negative, positive result for $\mathrm{HBc} A b$ and $\mathrm{HBs} \mathrm{Ab}$ ). Bedside ultrasound showed the presence of moderate peritoneal and
Table 1. Laboratory results

\begin{tabular}{|c|c|c|}
\hline Variable & Value on admission & Reference range \\
\hline CRP, $\mathrm{mg} / \mathrm{dL}$ & 9.45 & $<0.5$ \\
\hline Procalcitonin, $\mathrm{ng} / \mathrm{mL}$ & 0.3 & $<0.25$ \\
\hline Hematocrit, $\%$ & 27.8 & $41.0-50.0$ \\
\hline Hemoglobin, $\mathrm{g} / \mathrm{dL}$ & 8.9 & $13.0-18.0$ \\
\hline White blood cell count, $\times 10^{9} / \mathrm{L}$ & 10.49 & $4.0-11.0$ \\
\hline Differential count, $\%$ & & \\
\hline Neutrophils & 76.3 & $40-75$ \\
\hline Lymphocytes & 9.3 & $20-50$ \\
\hline Monocytes & 6.2 & $2-13$ \\
\hline Eosinophils & 7.5 & $0.0-7.0$ \\
\hline Platelet count, $\times 10^{9} / \mathrm{L}$ & 278 & $150-450$ \\
\hline Creatinine, $\mathrm{mg} / \mathrm{dL}$ & 0.54 & $0.6-1.2$ \\
\hline Aspartate aminotransferase, $\mathrm{U} / \mathrm{L}$ & 52 & $<45$ \\
\hline Alanine aminotransferase, $\mathrm{U} / \mathrm{L}$ & 36 & $<40$ \\
\hline Lactate dehydrogenase, $\mathrm{U} / \mathrm{L}$ & 312 & $30-200$ \\
\hline Haptoglobin $\mathrm{mg} / \mathrm{dL}$ & 265 & \\
\hline
\end{tabular}

right pleural effusion. Analyses of ascitic and pleural fluid revealed a high leukocyte count $\left(2.5 \times 10^{9} / \mathrm{L}\right.$ and $2.3 \times 10^{9} / \mathrm{L}$, respectively) with predominance of lymphocytes. Cytological examination, bacterial culture, acid-fast staining and polymerase-chain-reaction (PCR) testing for Mycobacterium tuberculosis on ascitic and pleural fluid resulted negative. A contrast-enhanced (CE) thoracic CT scan documented an enlarged right pulmonary hilar lymph node $(3.5 \mathrm{~cm})$, in the absence of lung lesions (Figure 1). An abdominal CT scan revealed the presence of peritoneal and omental thickening (omental cake) with peritoneal enhancement, matted, dilated bowel loops, and splenomegaly.

${ }^{\star}$ Correspondence to: Antonio Faraone, Internal Medicine Unit, San Giovanni di Dio Hospital, Florence, Italy, E-mail: antonio.faraone@uslcentro.toscana.it

Key words: tuberculosis, ascites, omental cake, peritonitis

Received: May 11, 2020; Accepted: June 03, 2020; Published: June 09, 2020 

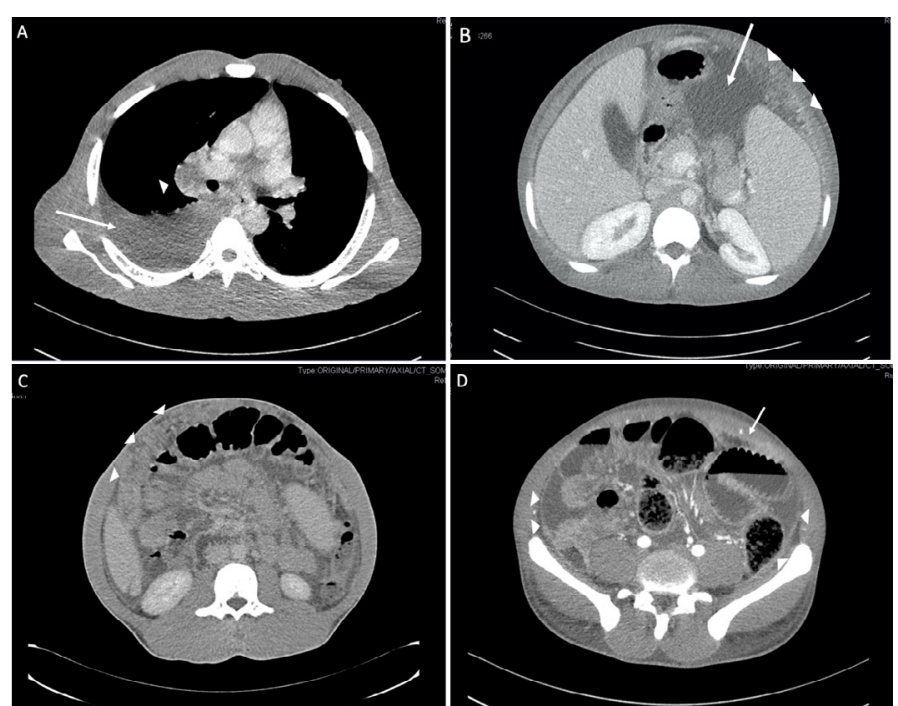

Figure 1. Contrast enhanced thoraco-abdominal CT scan. A: right pleural effusion (harrow) and enlarged right hilar limph node (arrowheads). B: omental thickening (arrowheads) and ascites (arrow); splenomegaly is present. C: extensive, omental thickening, displacing underlying bowel from the abdominal wall (omental cake, arrowheads). D: omental thickening (arrow), peritoneal enhancement (arrowheads), and matted, dilated bowel loops

Based on these findings, the patient underwent a fibrobronchoscopy with bronchoalveolar lavage (BAL) and endobronchial ultrasound transbronchial needle aspiration (EBUS/TBNA). The transbronchial needle biopsy of the right pulmonary hilar lymphadenopathy revealed the presence of necrotizing granulomas, while staining and PCR testing for tubercular bacilli on biopsy sample and BAL resulted negative. Considering the histological findings and clinical context, a presumptive diagnosis of TB with prevalent peritoneal involvement was made. The patient underwent a standard anti-tuberculous treatment (isoniazid, rifampin, ethambutol, and pyrazinamide for 2 months, followed by isoniazid and rifampin for 4 months), experiencing significant clinical, biochemical and radiological improvement after 2 months (disappearance of fever and abdominal pain, reduction of CRP level, decrease of peritoneal and right pleural effusion), and full recovery at the end of treatment. Mycobacterial cultures on ascitic and pleural fluid, BAL and lymph node biopsy resulted negative.

\section{Discussion}

Tuberculous peritonitis is an uncommon disease in low $\mathrm{TB}$ incidence countries, representing $4.9 \%$ of extrapulmonary TB cases in the United States, and most often affecting non-white race, foreignborn individuals $[1,2]$. This pathological condition is characterized by a subacute presentation, evolving over a period of several weeks to months; the main clinical features include systemic symptoms such as fever, weight loss, anorexia, malaise, associated with abdominal pain and ascites [3]. Diagnosis of tuberculous peritonitis in low TB incidence areas can be challenging, due to the rarity of the infection, its nonspecific clinical picture and paucibacillary nature. The disease should be suspected in patients with a consistent clinical picture and risk factors for TB. The index of suspicion should be higher in individuals with HIV infection, alcoholic liver disease, and chronic renal failure on continuous peritoneal dialysis [3]. In the present case, the patient was an immigrant from a high TB endemic country and, in addition, he was an alcohol abuser.

Ascitic fluid analysis in individuals with tuberculous peritonitis is typically consistent with an exudate, characterized by high protein level and high leukocyte count with predominance of lymphocytes [3]. The yield of acid-fast smear microscopy, PCR and culture testing for $M$. tuberculosis is quite low ( $<5 \%, 4-28 \%, 45-69 \%$, respectively) $[4,5]$; as a consequence, paracentesis is rarely diagnostic. Adenosine deaminase (ADA) level on ascitic fluid has been identified as a sensitive and specific tool for early diagnosis of tuberculous peritonitis [4]: unfortunately, in the present case we could not measure the ADA level, since the laboratory of our institution does not perform such assay.

CT imaging findings, though not specific, can aid the diagnosis of tuberculous peritonitis in the presence of a suggestive clinical history. The main findings consist of peritoneal effusion, nodules and diffuse thickening of peritoneum and omentum (the so-called "omental cake") with contrast-enhancement [6]. Other potential features include peritoneal omental mass, enlarged and necrotic mesenteric lymph nodes, mesenteric macronodules, splenomegaly. All these radiological signs pose problems in differential diagnosis from other peritoneal diseases, primarily malignancy (peritoneal carcinomatosis), and should prompt further investigations [6].

Definitive diagnosis of tuberculous peritonitis often requires a laparoscopic biopsy of peritoneum, or bioptic sampling of any other tissue potentially interested by the infection. If tuberculous bacilli are not identified on the biopsy specimen (by means of acid-fast staining, PCR, culture methods), diagnosis of TB can be supported by the demonstration of necrotizing or non-necrotizing granulomas on histological examination [4]. In the present case, a transbronchial lymph node biopsy was preferred to laparoscopy and peritoneal sampling, and a presumptive diagnosis of TB was made, based on the histological results and clinical context. The improvement of the patient obtained after the first 2 months of antituberculosis treatment offered a confirmation of the diagnosis.

\section{References}

1. Peto HM, Pratt RH, Harrington TA, LoBue PA, Armstrong LR (2009) Epidemiology of extrapulmonary tuberculosis in the United States, 1993-2006. Clin Infect Dis 49: 1350-1357.

2. Ben Ayed H, Koubaa M, Marrakchi C, Rekik K, Hammami F, et al. (2018) Extrapulmonary tuberculosis: Update on the epidemiology, risk factors and prevention strategies. Int J Trop Dis 1: 1-6.

3. Sanai FM, Bzeizi KI (2005) Systematic review: Tuberculous peritonitis - presenting features, diagnostic strategies and treatment. Aliment Pharmacol Ther 22: 685-700.

4. Lewinsohn DM, Leonard MK, LoBue PA (2017) Official american thoracic society/ infectious diseases society of america/centers for disease control and prevention clinical practice guidelines: Diagnosis of tuberculosis in adults and children. Clin Infect Dis 64: 1115-1119.

5. Dahale AS, Puri AS, Kumar A, Dalal A, Agarwal A, et al. (2019) Tissue Xpert ${ }^{\circledR}$ MTB RIF Assay in peritoneal tuberculosis: To be (done) or not to be (done). Cureus 11 e5009.

6. Mamlouk MD, vanSonnenberg E, Shankar S, Silverman SG (2011) Omental cakes: Unusual aetiologies and CT appearances. Insights Imaging 2: 399-408.

Copyright: (C2020 Faraone A. This is an open-access article distributed under the terms of the Creative Commons Attribution License, which permits unrestricted use, distribution, and reproduction in any medium, provided the original author and source are credited. 